\title{
Modeling of Plasmon-Enhanced Photoluminescence of Si Nanocrystals Embedded in Thin Silicon-Rich Oxinitride Layer
}

\author{
Z. ÉDES ${ }^{a, b, *}$, V. KŘÁPEK ${ }^{a}$ AND T. Š́KOLA ${ }^{b}$ \\ ${ }^{a}$ Central European Institute of Technology, Brno University of Technology, \\ Technická 10, CZ 61600 Brno, Czech Republic \\ ${ }^{b}$ Institute of Physics, Brno University of Technology, Technická 2, CZ 61669 Brno, Czech Republic
}

Plasmon-enhanced photoluminescence of silicon nanocrystals embedded in silicon-rich oxinitride thin film is calculated using finite-difference time-domain simulations. Emitters are represented as point-like dipoles and the photoluminescence enhancement is calculated depending on the emitter's position and polarization with respect to the plasmonic metal nanoparticle placed on top of the layer. We show that the photoluminescence enhancement is dominated by the excitation enhancement even for tuning the metal nanoparticle size to the emission wavelength.

DOI: 10.12693/APhysPolA.129.A-70

PACS: 73.20.Mf, 78.55.Qr, 42.50.Nn, 78.20.Bh

\section{Introduction}

Since the discovery of E.M. Purcell in 1946 it is known that photoluminescence (PL) can be strongly affected by the environment in which is the emitter placed [1]. The emerging field of plasmonics offered a good alternative for enhancing PL by coupling the emitter to localized surface plasmons (LSPs) of metallic nanoparticles (MNPs). This effect, known as plasmon-enhanced PL, is extensively studied in the recent years and the mechanisms of the enhancement are already known and well described [2-5]. By tuning the plasmon resonances of MNPs at the emission wavelength the emission rate of luminescent material can be significantly altered. Achieving the desired enhancement needs not only tuning of the LSP resonances, but also precise engineering of the MNP-emitter distance down to nanometer precision. For simple geometries and point-like emitters it is possible to calculate the enhancement analytically [6], but for most of the experiments numerical simulations are required to confirm the experimental results. This theoretical study is focused on the special case, when MNPs are placed on the substrate in which the randomly distributed emitters are embedded. It was motivated by the growing interest in light emitting silicon nanocrystals ( $\mathrm{NCs}$ ), which are commonly fabricated by thermal treatment of silicon monoxide or silicon-rich oxinitride films $[7,8]$.

\section{Methods}

Enhancement of the PL is the product of excitation enhancement $Q_{\text {exc }}$ and quantum efficiency (QE) enhancement $Q_{\mathrm{em}}[4]$ :

$$
I=Q_{\mathrm{exc}} Q_{\mathrm{em}} .
$$

The excitation enhancement is connected with the local

*corresponding author; e-mail: zoltan.edes@ceitec.vutbr.cz field enhancement by the MNP and scales with the ratio between the local electric field intensity with and without the MNP: $Q_{\mathrm{exc}}=|\mathbf{E}|^{2} /\left|\mathbf{E}_{0}\right|^{2}[3]$. QE enhancement is obtained from the ratio between the modified and the intrinsic $\mathrm{QE}$ of the excited emitter $Q_{\mathrm{em}}=\eta / \eta_{0}$. The intrinsic QE $\eta_{0}$ is defined as:

$$
\eta_{0}=\frac{\gamma_{\mathrm{r} 0}}{\gamma_{\mathrm{r} 0}+\gamma_{\mathrm{nr} 0}}
$$

where $\gamma_{\mathrm{r} 0}$ and $\gamma_{\mathrm{nr} 0}$ are the radiative and non-radiative decay rates of the transition from the excited state, respectively. Presence of MNPs changes the radiative decay rate $\gamma_{\mathrm{r}}$ and opens a new non-radiative decay channel $\gamma_{\mathrm{nr}}$ caused by absorption losses in metal, therefore the modified QE yields [4]:

$$
\eta=\frac{\gamma_{\mathrm{r}}}{\gamma_{\mathrm{r}}+\gamma_{\mathrm{nr}}+\gamma_{\mathrm{nr} 0}} .
$$
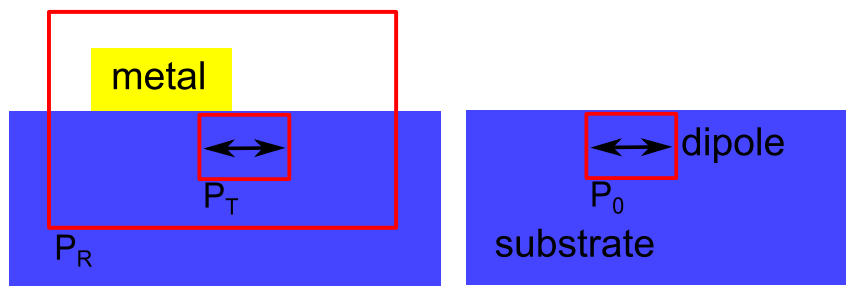

Fig. 1. A scheme of the simulation model for the decay rate modifications. The scheme shows the location of two monitor boxes calculating the power transmitted through them $\left(P_{\mathrm{R}}, P_{\mathrm{T}}\right)$ and the reference structure containing only the dipole without the metal particle (calculating $P_{0}$ ).

To calculate these modifications a simple classical model is used, where the emitter is represented as a pointlike dipole. The power emitted by the dipole $\left(P_{\mathrm{T}}\right)$ and the MNP-dipole complex $\left(P_{\mathrm{R}}\right)$ is calculated and consequently normalized to the power emitted by the dipole without the MNP $\left(P_{0}\right)$, yielding values $Z_{\mathrm{R}}$ and $Z_{\mathrm{T}}$, respectively (see Fig. 1). The physical meaning of these values is the following: $Z_{\mathrm{r}}$ represents the ratio between 
$\gamma_{\mathrm{r}}$ and $\gamma_{\mathrm{r} 0}$ and $Z_{\mathrm{t}}$ yields the ratio between $\gamma_{\mathrm{nr}}$ and $\gamma_{\mathrm{r} 0}$ Thus the QE enhancement can be expressed as

$$
Q_{\mathrm{em}}=\frac{Z_{\mathrm{R}}}{\eta_{0} Z_{\mathrm{T}}+1-\eta_{0}}
$$
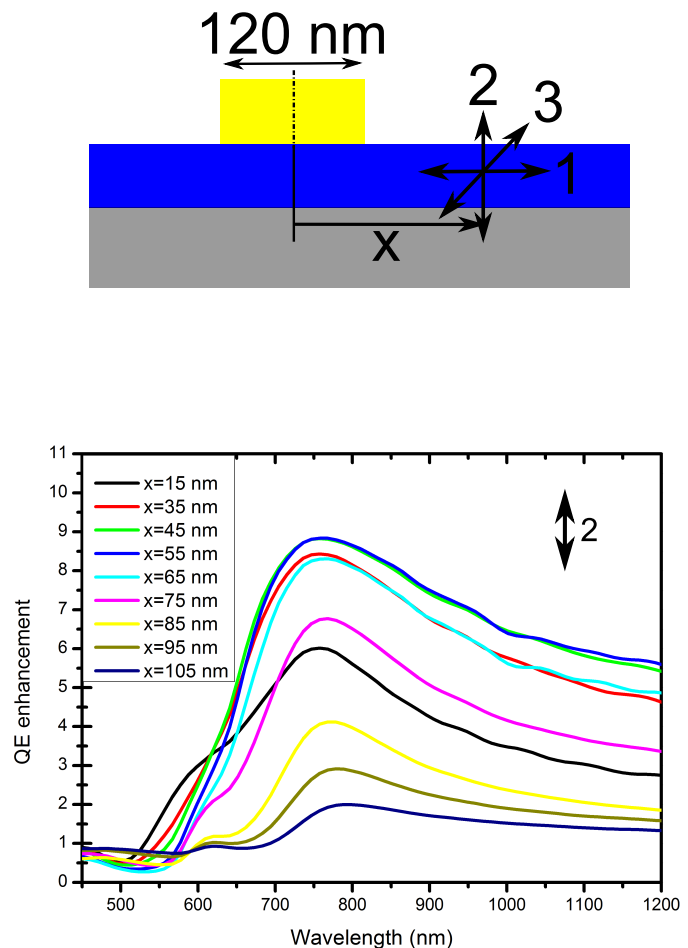

Fig. 2. The modified quantum efficiency for different horizontal distances from the metallic nanoparticle axis. Dipole polarization is indicated in each graph and the scheme reveals the simulation layout.
Calculations of $Z_{\mathrm{R}}$ and $Z_{\mathrm{T}}$ were performed numerically using finite-difference time-domain (FDTD) software Lumerical Solutions. Thickness of the oxide layer was set to $20 \mathrm{~nm}$ and $\mathrm{Si}$ was used as a substrate material (see Fig. 2). A $30 \mathrm{~nm}$ thick gold disks were used as MNPs on top of the thin layer. The dipole was placed in a fixed depth of $10 \mathrm{~nm}$. The simulations were performed for different horizontal distance between the MNP's axis and the dipole position and for three different dipole polarizations.

\section{Results and discussion}

The intrinsic QE of Si NCs is strongly dependent e.g. on preparation technique and the surrounding medium and varies from 0.01 up to 0.60 [9]. Therefore, during simulations we use a value with typical magnitude $\eta_{0}=0.1$. Similar variation is observable in the emission wavelength due to its strong dependence on nanocrystal size. Based on the published literature we expect emission around $800 \mathrm{~nm}$, which means the size of the MNPs
Equation (4) shows that using this model by knowing the intrinsic $\mathrm{QE}$ and by calculating $Z_{\mathrm{R}}$ and $Z_{\mathrm{T}}$ it is possible to determine QE enhancement.
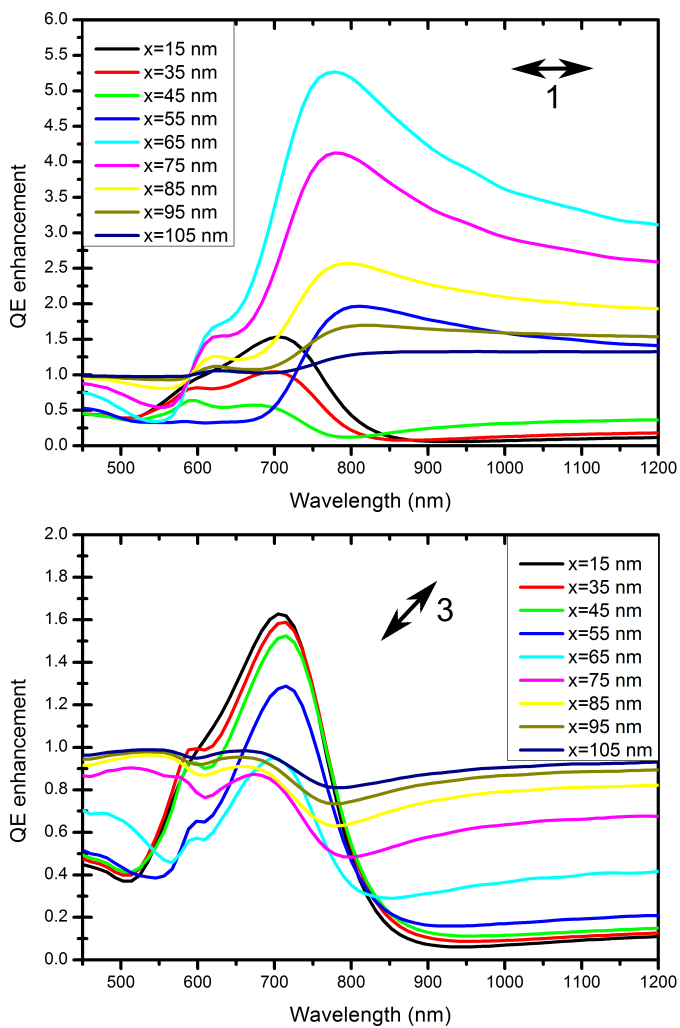
at this wavelength [8]. In order to find the ideal disk size we calculate the modified QE for dipole polarized perpendicularly to the MNP and placed in horizontal distance of $5 \mathrm{~nm}$ from the disk edge. It is apparent from Fig. 3 that the peak position of QE changes with the disk diameter. However, in the further simulations we choose diameter $d=120 \mathrm{~nm}$ as it matches best the expected emission wavelength of the NCs.

Figure 2 shows the simulated QE enhancement for three basic dipole polarizations at different positions. Value of $x$ denotes the horizontal distance of dipole from the disk main axis, which means that for distances smaller than $60 \mathrm{~nm}$ the dipole is situated below the MNP.

Results are showing that for different dipole orientation we got strongly distinct characteristics. For polarization 1 the PL of emitters laying below the MNP is quenched and for emitters which are not beneath the metal it is enhanced. Polarization 3 has opposite behavior but the enhancements factors are much lower and on the other hand the quenching is more pronounced than for polar- 


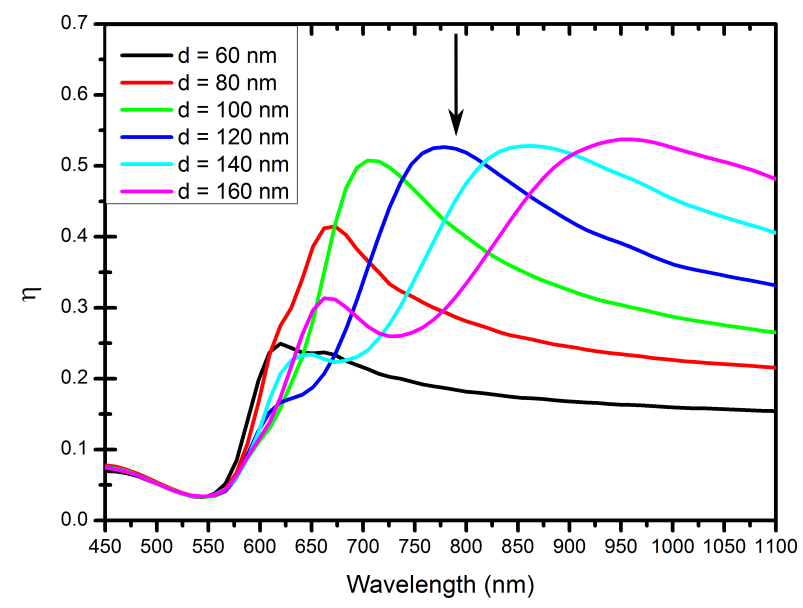

Fig. 3. The modified quantum efficiency for different metallic nanoparticle sizes with the chosen diameter marked with black arrow.

ization 1. Interestingly, for polarization 2 the PL is enhanced for all positions and also the enhancement factors are the highest.

Finally, the excitation enhancement is calculated using numerical simulations as well. The MNP is illuminated by a plane wave impinging perpendicularly on the substrate and the electric field is monitored at positions where the dipoles were previously placed. The excitation enhancement is evaluated for two commonly used laser wavelengths: $532 \mathrm{~nm}$ and $633 \mathrm{~nm}$. It is apparent from the Fig. 4, that although the size of MNPs was optimized to match the emission wavelength, at $532 \mathrm{~nm}$ we obtain the same enhancement factors for $Q_{\text {exc }}$ as for $Q_{\mathrm{em}}$. Moreover for excitation at $633 \mathrm{~nm}$ the excitation enhancement exceeds the emission enhancement by one order of magnitude.

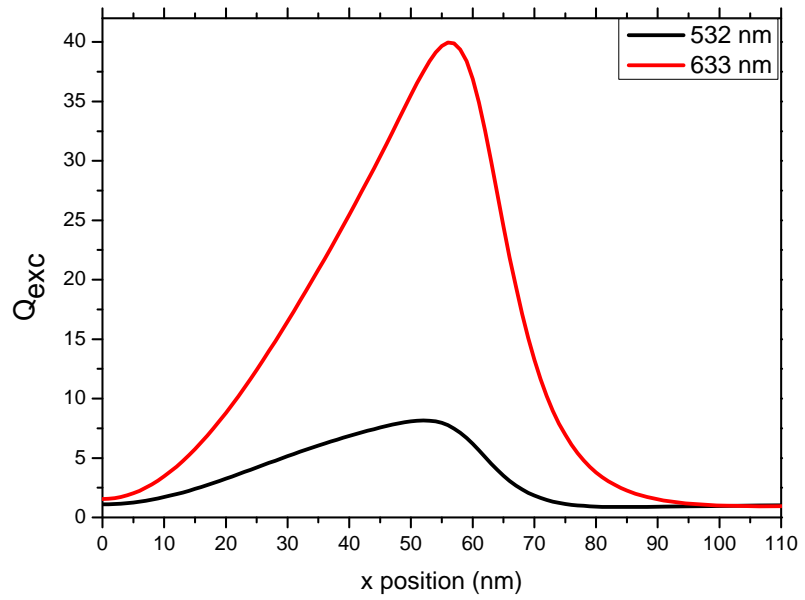

Fig. 4. Excitation enhancement in depth of $10 \mathrm{~nm}$ in the oxinitride layer for two wavelengths $532 \mathrm{~nm}$ (black line) and $633 \mathrm{~nm}$ (red line).

\section{Conclusions}

As the light emission from the Si NCs has random polarization the averaged emission enhancement should be considered from the 3 basic polarizations. The value of this enhancement reaches 3-5 fold enhancement for ideal distances from the MNP $(55-75 \mathrm{~nm})$. It is important to note that the emitter-MNP coupling is limited to small distances, as for distance $x=105 \mathrm{~nm}$ (45 nm from the MNP edge) yields almost negligible emission enhancements. Surprisingly, at the same distances the $Q_{\text {exc }}$ yields up to one order of magnitude higher values even if the size of MNPs is not optimized for excitation enhancement. Therefore, it will be more effective to tune the MNPs to get maximum excitation enhancement as this effect yields higher enhancement factors. Nevertheless, the overall PL enhancement for ideal distances reaches 2 orders of magnitude according to our calculations, but it is dominated by the excitation enhancement. Finally, from experimental point of view the emission directionality of MNPs should be also considered as it can play a significant role in the measured PL enhancement.

\section{Acknowledgments}

This work has been supported by the projects: European Regional Development Fund (CEITEC CZ.1.05/1.1.00/02.0068), EU 7th Framework Programme (Contract No. 286154 - SYLICA and 280566 UnivSEM), Technology Agency of the Czech Republic (Te01020233), Grant Agency of the Czech Republic (grant No. 15-21581S). V.K. was supported by European Social Fund (grant No. CZ.1.07/2.3.00/30.0005).

\section{References}

[1] E.M. Purcell, Phys. Rev. 69, 681 (1946).

[2] H. Atwater, A. Polman, Nature Mater. 9, 205 (2010).

[3] C.A. Orozco, J.G. Liu, M.W. Knight, Y. Wang, J.K. Day, P. Nordlander, N.J. Halas, Nano Lett. 14, 2926 (2014).

[4] L. Novotny, P. Bharadwaj, Opt. Expr. 15, 14266 (2007).

[5] A.M. Kern, O.J.F. Martin, Nano Lett. 11, 482 (2011).

[6] H. Mertens, A.F. Koenderink, A. Polman, Phys. Rev. $B$ 76, 115123 (2007).

[7] S. Kohli, J.A. Theil, P.C. Dippo, K.M. Jones, M.M. Al-Jassim, R.K. Ahrenkiel, C.D. Rithner, P.K. Dorhout, Nanotechnology 15, 1831 (2004).

[8] J. Goffard, D. Gerard, P. Miska, A.L. Baudrion, R. Deturche, J. Plain, Sci. Rep. 3, 2672 (2013).

[9] I. Pelant, Phys. Status Solidi A 208, 625 (2010). 